\title{
Type I Collagen C-Telopeptide Measurement
}

National Cancer Institute

\section{Source}

National Cancer Institute. Type I Collagen C-Telopeptide Measurement. NCI Thesaurus.

Code C82038.

The determination of the amount of the type I collagen C-telopeptide present in a sample. 